\title{
Unraveling the cell entry mechanisms of HSV: therapeutic potential?
}

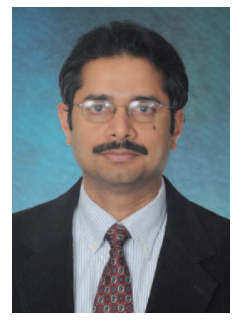

\author{
“... the HSV entry field has progressed so much in \\ the last two decades that we can explain many \\ unique aspects that make HSV an outstandingly \\ successful invader of human cells."
}

\section{Deepak Shukla}

Department of Ophthalmology \& Visual Sciences, University of Illinois at Chicago, Chicago, IL, USA and

Department of Microbiology \& Immunology, University of Illinois at Chicago, Chicago, IL, USA -dshukla@uic.edu

Entering into a host cell does not appear to be a great challenge for HSV. As a matter of fact, it is extremely hard to find a cultured cell type that does not allow HSV entry [1], which has been a puzzling topic for many scientists. With all the latest advances in the field, it may seem like that we finally have all the answers to explain the 'promiscuity' of HSV with human cells. Indeed, the HSV entry field has progressed so much in the last two decades that we can explain many unique aspects that make HSV an outstandingly successful invader of human cells [2]. However, the puzzle is far from being completely solved. Virtually every year we hear about new receptors for HSV, and it seems that this list will keep growing for the foreseeable future. Does this mean that HSV can bind to and interact with virtually all proteins expressed on cell surfaces? Is the virus capable of 'enticing' even the cytoplasmic proteins to conveniently appear on the cell surface to demonstrate a 'receptor-style' binding with the virus? It would not be at all surprising if this turned out to be the case! A cell biologist colleague recently told me that HSV entry scientists would love to change the "very biology of cell biology". Of course, this colleague thought that we are the ones challenging the conventional wisdom of cell biologists, but in fact it is the virus doing the challenging!

So, does the virus that my research focuses on really defy the conventional wisdom of the cell biologists? The truth is that this successful dsDNA virus, with an approximate 150$\mathrm{kDa}$ genome and a dozen glycoproteins on its envelope, might be defying the established wisdom of all kinds of biologists [1]. Even the immunologists, who have been trying to use everything they can in the field of immunity to find an effective vaccine against the virus have tailed thus far [3]. In fact, it seems that the virus cannot possibly be controlled by conventional vaccines, since most infected individuals already have the neutralizing antibodies, but many still keep redeveloping symptoms, and virtually all of them periodically shed the virus through their bodily fluids [4]. So, what is going on here? Why can we not eradicate this virus? To address this it is necessary to explain why it is important to focus on cell entry, and why blocking entry and combining it with the development of immunity may be our ultimate weapon against the seemingly unstoppable spread of HSV.

6. ...this successful dsDNA virus ... might be defying the established wisdom of all kinds of biologists."

HSV-1 infection is one of the most common communicable diseases among humans. Facial herpes causes 500,000 cases of herpes labialis annually, affecting the lips, mouth, gums or areas immediately surrounding the lips [1-2]. Ocular HSV-1 infections lead to stromal keratitis, blepharitis and retinitis [4]. In addition, immunocompromised hosts can suffer from HSV encephalitis and visceral infections, including, but not limited to, pneumonitis, esophagitis and hepatitis. Therefore, the virus is capable of causing significant health problems in humans, and it all starts with a HSV particle finding a way to enter into a host cell, usually a mucosal epithelial cell. The virus actually starts priming cells for an incoming infection even before its entry into the cell. It appears that the virus binds to the filopodia of cells to facilitate its unidirectional transport to efficiently reach cell bodies for entry [5]. The filopodia are usually present on the surfaces of cells, forming the leading edges of the migrating

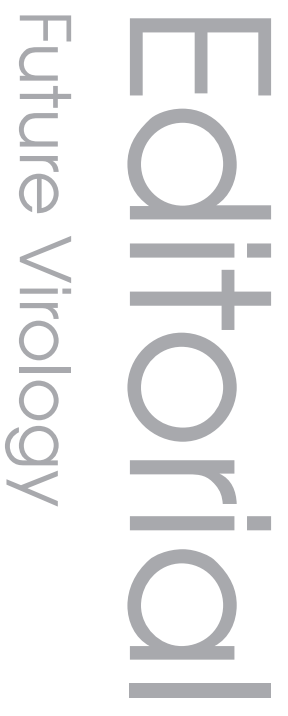

\section{Keywords}

- envelope glycoproteins - heparan sulfate $=$ herpes simplex virus $=$ microbicide " vaccine " virus entry

Future Medicine part of 
tissue layers such as those found during wound healing. It has been demonstrated that incoming HSV-1 virions can themselves induce filopodia formation by the cells.

Attachment to filopodia and to cells in general is mediated by HSV-1 envelope glycoproteins $\mathrm{gB}$ and $\mathrm{gC}$. Both bind heparan sulfate (HS) moieties present on the cell surface, and $\mathrm{gB}$ appears to be most critical for viral movement along filopodia in a process called 'surfing' [6]. This process alone is not sufficient for infection though. Actually, viral interactions with HS may be an ideal stage for the development of new prophylactics, since at this stage the virus has still not entered the cell. The cell body expresses entry receptors, which the virus requires for its penetration into cells, and some of the most-studied receptors are those for envelope glycoprotein $\mathrm{gD}$. The gD receptors belong to three different families of cell-surface receptors [1]. Herpes virus entry mediator belongs to the family of TNF receptors, whereas nectin-1 is a well-studied member of the immunoglobulin superfamily. Interestingly, some rare modifications within $\mathrm{HS}$, which generate 3 - $\mathrm{O}$-sulfated $\mathrm{HS}$ give rise to the final member of the $\mathrm{gD}$ receptor family [7]. Once again, as an example of unconventional biochemistry, $\mathrm{gD}$ not only binds to two unrelated protein receptors (herpes virus entry mediator and nectin-1) with equal affinity, but also shows a virtually identical binding affinity, for the polysaccharide 3-O-sulfated HS found on cell surfaces, and in each case, its interaction yields an identical result - that is, to trigger viral envelope fusion with a host cell membrane. One $\mathrm{gD}$, three completely unrelated host cell receptors, and the net result is viral penetration into the host cell [8].

\footnotetext{
"Recent advances in the HSV entry field have created a tremendous new opportunity for the development of new prophylactics and/or therapeutics against HSV."
}

Or perhaps not! The story of herpes entry is not that simple. The binding of $\mathrm{gD}$ to one of its receptors is supposed to trigger the assembly of the membrane fusion complex, which requires participation from additional glycoproteins, $\mathrm{gB}$ and $\mathrm{gH}-\mathrm{gL}[1]$. The glycoprotein $\mathrm{gB}$, a fusogen, has its own unique receptors. The story gets even more complicated at this point, since the exposure of HSV-1 to cells somehow triggers enhanced localization and an unusual presence of a normally cytoplasmic protein, non-muscle myosin heavy chain-IIA, to the plasma membrane [9]. In a poorly understood mechanism, $\mathrm{gB}$ binds to non-muscle myosin heavy chain and uses it for its fusogenic activity during HSV-1 entry. The other two unrelated gB receptors include paired immunoglobulin-like type 2 receptor- $\alpha$ and myelin-associated glycoprotein [1]. Knocking down either a $\mathrm{gD}$ receptor or a $\mathrm{gB}$ receptor inhibits virus from entering the cells. So, what about gH-gL? It appears that gH may bind integrins [10], although this interaction may not be essential for entry. However, interaction with integrins is noteworthy since many other herpesviruses also interact with integrins. The latter brings attention to at least two different modes of HSV-1 entry. The more conventional, fusion of viral envelope with plasma membrane of the host cells has been shown to be limited to certain cell types, while many others allow entry via a clathrin or caveolin-independent endocytic mode that mimics phagocytosis [5]. This should perhaps not be considered too unusual, however, for a virus that seems to do many other unconventional things. Integrins tend to signal entry via endocytosis/phagocytosis and thus, may form a critical component of viral entry through the endocytic route [11].

Whether via endocytosis or otherwise, the virus needs to find ways to fuse its envelope either with the membrane of a vesicle or the plasma membrane to release the capsid and tegument proteins into the cytoplasm for the start of new virus production. Viral transcription and replication occur in the nucleus [2]. The same viral fusion machinery that mediates fusion at the plasma membrane is also required for fusion with the vesicular membrane since, irrespective of the mode of entry, $\mathrm{gB}, \mathrm{gD}$ and $\mathrm{gH}-\mathrm{gL}$ are essential for entry into any cell type. While gB represents a unique fusogen, $\mathrm{gH}$ may also have the qualities of a fusogen [1]. However, the reasons why HSV requires two fusogens, and why neither of these belong to the families of traditional fusogens, are poorly understood issues, and add to the complexity of the virus infection process.

Recent advances in the HSV entry field have created a tremendous new opportunity for the development of new prophylactics and/or therapeutics against HSV. A slew of new reagents and assays are now available that can greatly enhance our ability to screen new compounds, small molecules and peptide libraries for entry-inhibiting activities. These include the availability of recombinant viruses that express reporter enzymes or 
fluorescent probes, recombinant cell types that develop color to mark viral entry, and cell-based, virus-free assays that can be used to mimic membrane fusion during viral entry and cell-to-cell fusion during viral spread, as well as fluorescencebased virus attachment assays that can make high-throughput screening highly feasible [5,7,12].

\section{"One gD, three completely unrelated host cell receptors, and the net result is viral penetration into the host cell."}

These recent advances have also identified new targets for drug development, such as HS. Interactions with HS are crucial for entry and, therefore, there is a strong potential for the development of effective microbicides by targeting HS. Lactoferrin, an iron-binding glycoprotein found in many exocrine fluids, including milk, saliva, tears and vaginal secretions, blocks HSV entry by interfering with viral interactions with HS [13]. Along the same lines, we have recently demonstrated that small peptides that block HS can prevent HSV-1 from infecting murine corneas [14]. Likewise, the small molecule surfen binds HS and blocks HSV entry [15]. Clearly, targeting HS has the added advantage of a broadspectrum effect since many other pathogenic viruses and bacteria bind HS. Apart from HS, antibodies against the receptors for envelop glycoproteins $\mathrm{gB}$ and $\mathrm{gD}$ or receptor downregulation by RNAi have also been very effective in blocking entry.

Glycoprotein targeting, although very promising, has not been successfully utilized as an antiHSV treatment. Given the inability of current therapies to ensure total viral clearance from the body, combined with the emergence of resistant strains against currently popular therapeutics such as acyclovir, it would be greatly advantageous to expand antiviral options to include entry; especially since the entry receptors may be equally important for the membrane fusion needed for cell-to-cell spread. It is the latter that allows HSV to escape from neutralizing antibodies and renders them less effective in controlling the infection. By targeting HSV glycoproteins $\mathrm{gB}, \mathrm{gD}, \mathrm{gH}$ and $\mathrm{gL}$, the viral infection could be halted at the binding, fusion and entry stages, which would therefore mean that subsequent steps would not take place. By designing small molecules with high binding affinity to specific domains of the entry glycoproteins, host and virus interactions will be disrupted and entry will be inhibited. Fortunately, crystal structures are now available to guide the engineering of small-molecule inhibitors against $\mathrm{gB}$ or $\mathrm{gD}[16,17]$.

To sum up, HSV transmission has been unstoppable so far and it is unlikely that a conventional vaccine will be able to provide sterile immunity against the virus. In lieu of an effective vaccine, targeting virus entry for prevention and/or treatment is an excellent alternative, and one that remains practically untapped for HSV. An ideal prophylaxis would incorporate a unique microbicide that prevents entry while simultaneously providing a platform for antigen presentation leading to the development of immunity. While no such microbicide exists currently, vaccine immunologists and HSV entry scientists need to combine their expertise and develop a 'microbivac' that will eventually help eliminate HSV completely. Until then, while soulmates may not, herpes will keep sharing its life with its hosts forever.

\section{Financial \& competing interests disclosure \\ The author's research is supported by the NIH grants A1057860, A1081869 and EY01792 (core grant). The author has no other relevant affiliations or financial involvement with any organization or entity with a financial interest in or financial conflict with the sub- ject matter or materials discussed in the manuscript apart from those disclosed. \\ No writing assistance was utilized in the production of this manuscript.}

\section{References}

1. Karasneh GA, Shukla D. Herpes simplex virus infects most cell types in vitro: clues to its success. Virol. J. 8, 481 (2011).

2. Akhtar J, Shukla D. Viral entry mechanisms: cellular and viral mediators of herpes simplex virus entry. FEBS J. 276, 7228-3726 (2009).

3. Johnston C, Koelle DM, Wald A. HSV-2: in pursuit of a vaccine. J. Clin. Invest. 121, 4600-4609 (2011).

4. Kaufman HE, Azcuy AM, Varnell ED et al. HSV-1 DNA in tears and saliva of normal adults. Invest. Ophthalmol. Vis. Sci. 46, 241-247 (2005).

5. Clement C, Tiwari V, Scanlan PM et al. A novel role for phagocytosis-like uptake in herpes simplex virus entry. J. Cell. Biol. 174, 1009-1021 (2006).

6. Oh MJ, Akhtar J, Desai P et al. A role for heparan sulfate in viral surfing. Biochem. Biophys. Res. Commun. 391, 176-181 (2010).

7. Shukla D, Liu J, Blaiklock P et al. A novel role for 3-O-sulfated heparan sulfate in herpes simplex virus 1 entry. Cell 99, 13-22 (1999).
8. Spear PG, Eisenberg RJ, Cohen GH. Three classes of cell surface receptors for alphaherpesvirus entry. Virology 275, 1-8 (2000).

9. Arii J, Goto H, Suenaga T et al. Non-muscle myosin IIA is a functional entry receptor for herpes simplex virus-1. Nature 467, 859-862 (2010).

10. Parry C, Bell S, Minson T et al. Herpes simplex virus type 1 glycoprotein $\mathrm{H}$ binds to alphavbeta3 integrins. J. Gen. Virol. 86, 7-10 (2005). 


\section{Editorial Shukla}

11. Margadant C, Monsuur HN, Norman JC et al. Mechanisms of integrin activation and trafficking. Curr. Opin. Cell Biol. 23, 607-614 (2011).

12. O'Donnell CD, Shukla D. A novel function of heparan sulfate in the regulation of cellcell fusion. J. Biol. Chem. 284, 2965429665 (2009).

13. Marchetti M, Trybala E, Superti F et al. Inhibition of herpes simplex virus infection by lactoferrin is dependent on interference with the virus binding to

glycosaminoglycans. Virology 318, 405-413 (2004).

14. Tiwari V, Liu J, Valyi-Nagy T et al. Anti-heparan sulfate peptides that block herpes simplex virus infection in vivo. J. Biol. Chem. 286, 25406-25415 (2011).

15. Schuksz M, Fuster MM, Brown JR et al. Surfen, a small molecule antagonist of heparan sulfate. Proc. Natl Acad. Sci. USA 105, 13075-13080 (2008).

16. Heldwein EE, Lou H, Bender FC et al. Crystal structure of glycoprotein B from herpes simplex virus 1. Science 313, 217-220 (2006).

17. Di Giovine P, Settembre EC, Bhargava AK et al. Structure of herpes simplex virus glycoprotein D bound to the human receptor nectin-1. PLoS Pathog. 7(9), e1002277 (2011). 